\title{
Fingerprint Recognition with Edge Detection and Dimensionality Reduction Techniques.
}

\author{
Ratiporn Chanklan ${ }^{*}$, Kedkarn Chaiyakhan, Anusara Hirunyawanakul, Kittisak Kerdprasop, Nittaya Kerdprasop \\ Data Engineering Research Unit, School of Computer Engineering, Suranaree University of Technology, \\ Nakhon Ratchasima 30000 Thailand.
}

*Corresponding Author: arc_angle@hotmail.com

\begin{abstract}
At present fingerprint recognition has been used widely, such as an authentication means of mobile phone usage and a monitoring for working hours. But the recognition performance of existing system low. We thus propose techniques to improve the recognition. We notice that edge detection techniques applied to the fingerprint images can enhance the quality of images and cause the improvement in image recognition We thus study the four edges detection techniques: sobel, prewitt, robert and canny. For faster classification we also apply two dimensionality reduction techniques: principal component analysis and linear discriminant analysis. Then, we identify fingerprint images with the algorithm support vector machine using linear kernel function. Experimental results showed that the pre-processing fingerprint images using canny edge detection with principal component analysis can increased the recognition rate from $64.3 \%$ to $88 \%$. On using canny edge detection with linear discriminant analysis, the fingerprint image recognition can be improved from $73.8 \%$ to $88 \%$
\end{abstract}

Keywords: fingerprint, edge detection, support vector machine, principal component analysis, linear discriminant analysis

\section{Introduction}

The combination of biological, medical, and computer technologies can be used to identify a person from his/her unique feature. Individual can be automatically identified by a comparison of such feature to the one that has been stored in the database. The system to authenticate or identify a person is called a biometric system. The physical characteristics of the people do not change over time but the physical behaviors may have changed. Thus identifying the person by physical characteristics is more reliable than the use of physical behavioral. At present fingerprint identification used to access smart devices such as cellular phones. The fingerprint biometric system is however not secure enough because the accuracy is less than a hundred percent. In the past, many researchers proposed edge detection techniques to be used to enhance the recognition. The recognition performance has been improved by the application of wavelet transform with prewitt edge detection $^{(1)}$. The edge detection with gray level watershed approach makes faster data classification and better performance $^{(2)}$. Edge detection is one important image processing techniques to recognize the fingerprint. It influences the image extraction and affects the matching of images. The algorithm should be chosen according to the characteristics of the image for the perfect detection ${ }^{(3)}$.

We thus study a variety of edge in detection techniques to enhance recognition rate of the fingerprint images. However, fingerprint images obtain from each individuals will look very similar and make the correct classification very difficult. Therefore, we have separate elements of images. The element is maintained major characteristics of fingerprint images. We separate element of image by applying four edges detection methods: sobel, prewitt, robert, and canny. Edge detection is expected to make a fingerprint images look clear. Then we apply two dimensionality reduction techniques: principal component analysis and linear discriminant analysis. Dimensionality reduction is applied to make a classification faster and save a memory. Then dimensionality reduced image data are fed into the algorithm that is used to perform the classification task. We use the support vector machine with linear kernel and then compare the performance of each model. 


\section{Theories}

\subsection{Edge detection}

Edge detection ${ }^{(4)}$ is to find line around the object in the image. When we know line around the object, we can calculate the area (size) or recognition type of the object. However, finding correct image edge detection perfect not an easy task. In particular, finding edge of image with low quality or uneven light is even harder. Edges can be detected by the difference of light intensity from one point to another point. If there is much difference of light intensity, the edges can be outlined clearly. If the difference of light intensity is low the edges are not clear. Edge detection techniques can be divided into two main groups: Gradient method and Laplacian method.

In this study, we use four gradient methods: sobel edge detection, prewitt edge detection, robert edge detection, and canny edge detection.

\subsubsection{Sobel edge detection}

Sobel $^{(4)}$ is used to find change of colors in image between object and background. The gradient value in each band is calculated by convolution image with filter of size $3 \times 3$. The result applying Sobel edge detection is show in Fig. 1.

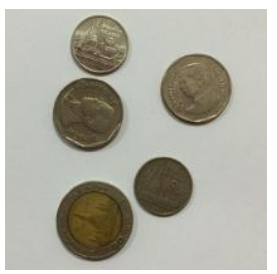

(a) Original image

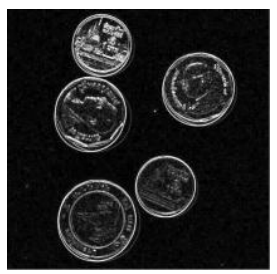

(b) Sobel edge detection image

Fig. 1. Demonstration of Sobel edge detection

\subsubsection{Prewitt edge detection}

Prewitt $^{(4)}$ performs edge detection by calculating gradient vector of each point on the original image. The higher gray level intensity shows border between object and background. Gradient is calculate by filter of size 3X3. Example of Prewitt edge detection is show in Fig. 2.

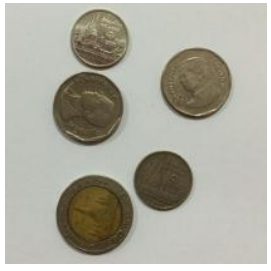

(a) Original image

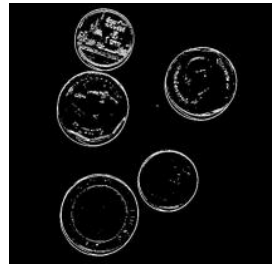

(b) Prewitt edge detection image
Fig. 2. Illustration of Prewitt edge detection

\subsubsection{Robert edge detection}

Robert $^{(4)}$ edge detection technique is similar to the Sobel edge detection. But use filter at smaller size of 2X2. Example of applying the Robert edge detection techniques is show in Fig. 3.

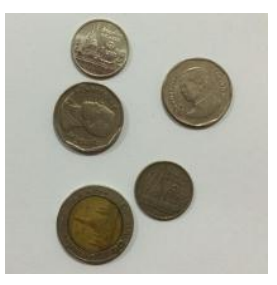

(a) Original image

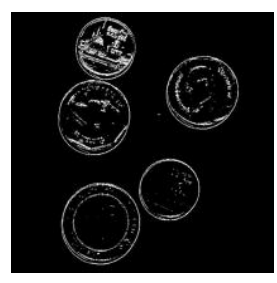

(b) Robert edge detection image
Fig. 3. Robert edge detection form samples image

\subsubsection{Canny edge detection}

Canny $^{(4)}$ edge detection applies adjustments to smooth image with Gaussian filter to remove noise in the image. It makes a better edge finding. Then calculate magnitude size and orientation of gradient. The next step is to use non-maxima suppression with Gradient magnitude to make the edges thinner. Finally use double thresholding algorithm to identify edge pixels and connect consecutive edges. Demonstration of Canny edge detection to the sample image is shown in Fig. 4.

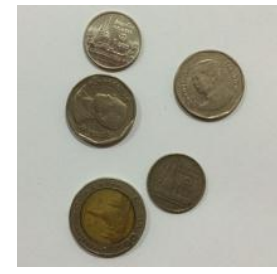

(a) Original image

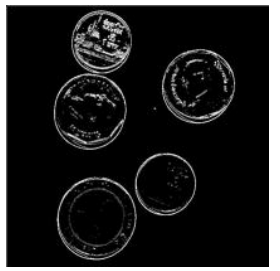

(b) Canny edge detection image
Fig. 4. Example of applying Canny edge detection 


\subsection{Dimensionality Reduction}

Dimensionality reduction is an action to replace original data with a new set of compact data. It reduces memory usage and uses less time for classification. Classification or recognition over a set of compact data should produce the same result as or equivalent classifying the original data.

In this study, we use two dimensionality reduction techniques: principal component analysis and linear discriminant analysis.

\subsubsection{Principal Component Analysis (PCA)}

$\mathrm{PCA}^{(5,6)}$ is the technique of multivariate data analysis without segmenting variables. It is commonly used to reduce size of the matrix of variables to smaller size appropriate for the further analysis of data. PCA will create a new variable, which is made up of variable or the variance of a combination of original variables. Finding relations among image normally uses the matrix of covariance from image data to build an Eigen faces instead of the vector (Eigenvector).

\subsubsection{Linear Discriminant Analysis (LDA)}

$\operatorname{LDA}^{(7)}$ is a technique used for supervised learning. It is commonly used for dimensionality reduction over data variables and also for classifying data. It uses a function to project data onto a subspace in such a way that data coming from different classes are well separated, and data from the same class are formed closer together to allow easy classification. It considers a distribution within group and distribution between the groups. LDA can identify picture that are affected by factors such as lights and shooting characteristics.

\subsection{Support Vector Machine (SVM)}

$\mathrm{SVM}^{(8,9)}$ is a process of selecting the optimal model for inducing the patterns. Support vector machine is popular in pattern recognition and data classification. Support vector machines for classification use optimal hyperplane in classifying data. Hyperplanes can be created in various ways. But therewill be the one optimal hyperplane that can maintain the greatest distance between two groups. The optimal hyperplane can be found by locating the support vector that is used as representative of the entire data set. This support vector is used to divide the data by taking a plane that can separate the two datagroups as much as possible. Then find a plane with maximum margin and assume that plane a suitable one for classification.

We assume a set of $\mathrm{n}$ data points, $\left(x_{i}, y_{i}\right), \ldots,\left(x_{n}, y_{n}\right)$ when $x \in R^{m}, y \in\{-1,1\}$. When $\mathrm{m}$ is dimension, $\mathrm{x}$ is data input, and y is class -1 or +1 . Creating a plane to split data can be calculated using Equations 1 and 2.

$$
\begin{aligned}
& \left(w * x_{1}\right)+b>0 \text { where } y_{i}=+1 \\
& \left(w * x_{2}\right)+b<0 \text { where } y_{i}=-1
\end{aligned}
$$

These equations classify data into either a class +1 or -1 , depending on the data attribute value, weight $(w)$, and bias(b). Example of creating a hyperplane to gain a maximum margin is shown in Fig. 5.

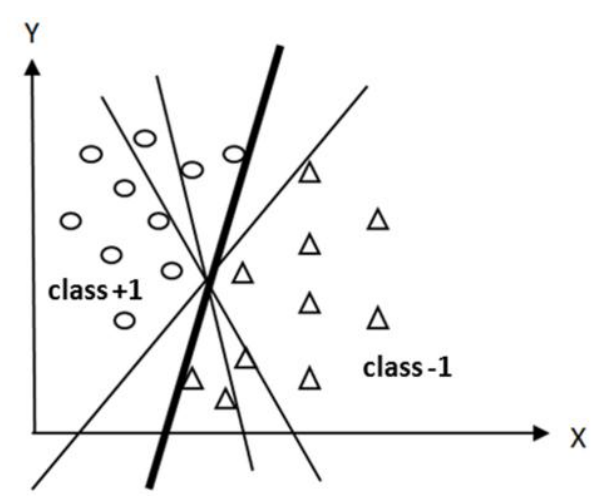

(a) Find hyperplane

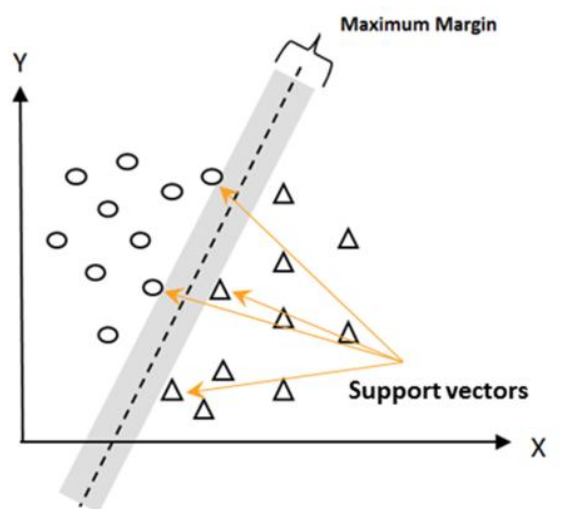

(b) Optimal hyperplane

Fig. 5. Optimal hyperplane for classification 


\section{Research Process}

This research used Fingerprint data set from http://fvs.sourceforge.net/download.html. It contains 168 fingerprint images of all 21 fingers; 1 finger has 8 images with a resolution of $80 \times 80$ pixels (6400 pixels).

In this study edges detection and dimensionality reduction have been applied to improve accuracy and to speed up the recognition. The process of experimentation is shown in Figure 6.

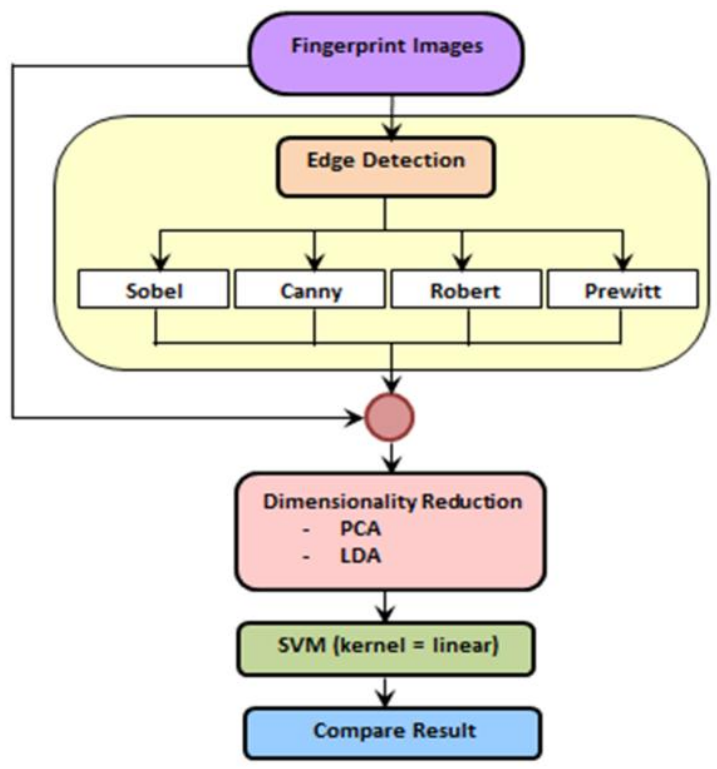

Fig. 6. The process of fingerprint recognition.

The first step of our comparative study is the application of 4 edges detection methods: sobel, prewitt, robert and canny. The implementation uses matlab program and python language with spyder editor to convert images data to array. Color in each pixel is convert to array 1 dimensional array. Then we apply dimensionality reduction techniques. In the last step, we use support vector machine algorithm to classify image data.

\section{Experimental Results}

This research measures the performance of the model by comparing the accuracy of the data classification with support vector machine algorithm. The experimental results before and after applying the edges detection techniques are shown in table 1, 2 and 3. Examples of fingerprint images obtained from each edge detection technique are illustrated in Fig. 7.
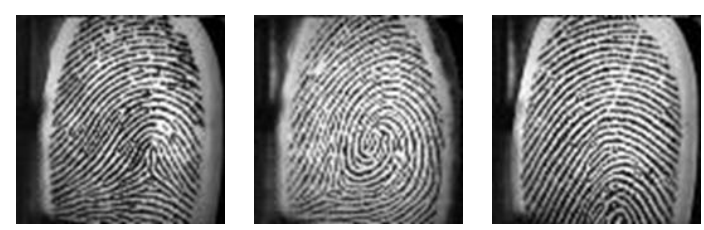

(a) Original fingerprint image
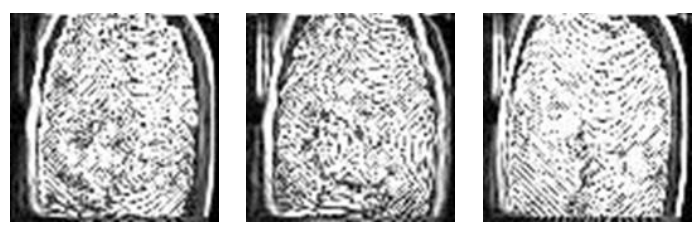

(b) Fingerprint image after applying sobel edge detection
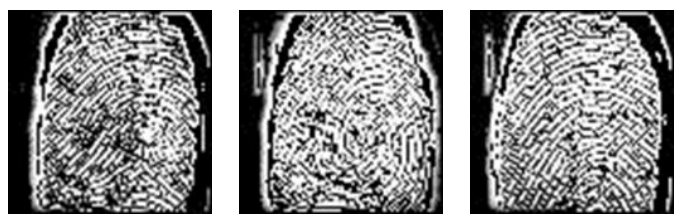

(c) Fingerprint image after applying canny edge detection.
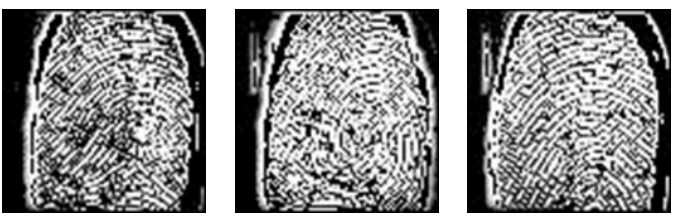

(c) Fingerprint image after applying canny edge detection
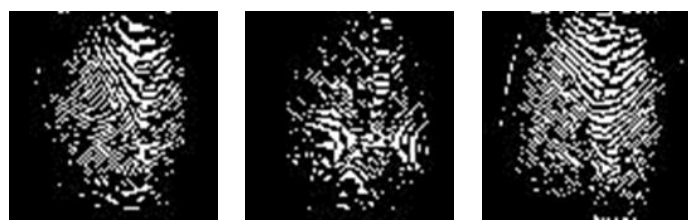

(d) Fingerprint image after applying robert edge detection.
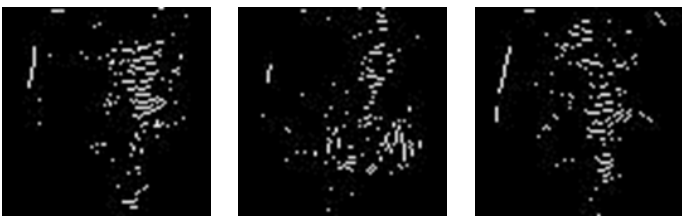

(e) Fingerprint image after applying prewitt edge detection.

Fig. 7. Fingerprint image samples. 


\subsection{Fingerprint Recognition Accuracy of Non-edge versus Edges Detection}

We split data into two datasets: training data containing 126 images and test data consisting of 42 images. Then perform classification using support vector machine algorithm with linear kernel. The classification accuracy is $52.4 \%$. This is the base line for comparison because it is the classification performance of the original image data. After applying edges detection: sobel, canny, and perwitt the classification accuracy increase.

Table 1. Before applying Dimensionality Reduction

\begin{tabular}{|c|c|}
\hline edge detection & accuracy \\
\hline Sobel & $85.7 \%$ \\
Canny & $71.4 \%$ \\
Robert & $38.1 \%$ \\
Prewitt & $52.4 \%$ \\
Non- edge & $52.4 \%$ \\
\hline
\end{tabular}

\subsection{Recognition Accuracy of Full Feature Fingerprint versus Dimensionality Reduction}

Table 2. Before applying edge detection

\begin{tabular}{|c|c|c|}
\hline Dimension reduce & component & accuracy \\
\hline LDA & 32 & $73.80 \%$ \\
PCA & 8 & $64.30 \%$ \\
\hline
\end{tabular}

When we use dimensionality reduction techniques, the classification accuracy increase significantly. LDA can increases accuracy from $52.4 \%$ to $73.80 \%$ and PCA increases accuracy from $52.4 \%$ to $64.30 \%$.

\subsection{Accuracy Improvement with Edge Detection and Dimensionality Reduction}

Table 3. After applying LDA with edge detection

\begin{tabular}{|c|c|c|}
\hline edge detection & component & accuracy \\
\hline Sobel & 32 & $88 \%$ \\
Canny & 32 & $71.4 \%$ \\
Robert & 16 & $40.5 \%$ \\
Prewitt & 32 & $45.2 \%$ \\
\hline
\end{tabular}

Table 4. After applying PCA with edge detection

\begin{tabular}{|c|c|c|}
\hline edge detection & component & accuracy \\
\hline Sobel & 7 & $88 \%$ \\
Canny & 10 & $83.3 \%$ \\
Robert & 6 & $52.5 \%$ \\
Prewitt & 16 & $71.4 \%$ \\
\hline
\end{tabular}

We have shown results before and after edge detection with two dimensionality reduction technique: principal component analysis (PCA) and linear discriminant analysis (LDA), as a graph in Fig. 8.

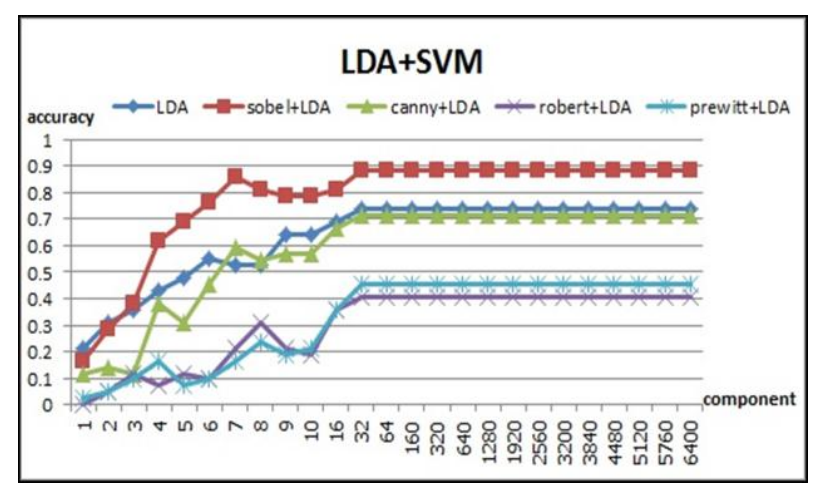

(a) This graph shows the accuracy with the components by edges detection and LDA.

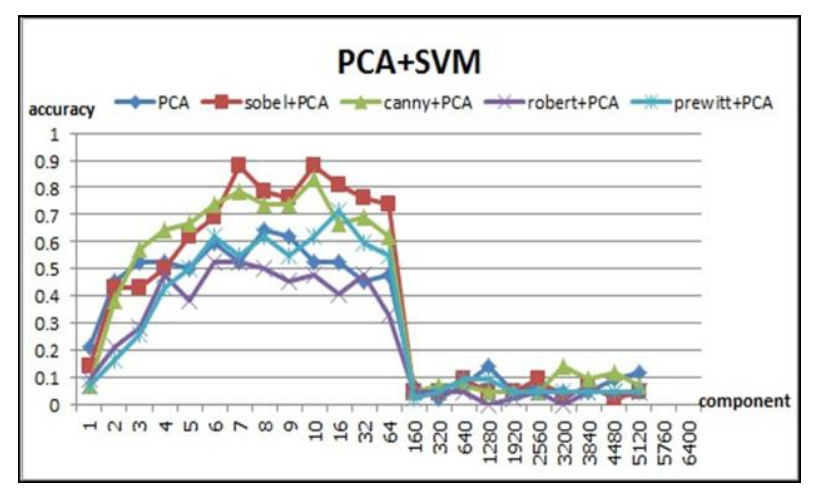

(b) This graph shows the accuracy with the components by edges detection and PCA.

Fig. 8. Graph shows all results. 
Fig. 8 is a graph showing the accuracy in accordance with the number of components. We are interested in the most accuracy model by comparing between edge detection method and non-edge detection method. Non-edges detected LDA + SVM gives the most accurate model at 73.8\%. After using sobel edge detection + LDA + SVM, model's accuracy increases to $88 \%$. Non-edge detected + PCA + SVM has the highest accuracy at $64.3 \%$. After using sobel edge detection + PCA + SVM the accuracy increase to $88 \%$. The accuracy is increased after applying edge detection because the fingerprint images can be noticed clearly. It can be seen from examples in Fig. 7 that the sobel edge detection technique converts prominent ridges into gray scale that can facilitate the classification algorithm.

\section{Conclusions}

The fingerprint recognition technology is widely used in many real-life applications such as the access to mobile devices, border control, entering building, and so on. Adopting fingerprints for identification and authentication is, however, still inaccurate. We propose in this paper the improvement of fingerprint image recognition through the use of edge detection and dimensionality reduction techniques. We use four edge detection methods: sobel, prewitt, robert, and canny. We apply dimensionality reduction techniques to help faster identification using two techniques: principal component analysis and linear discriminant analysis. Then classify fingerprint image with support vector machine using linear kernel. In a series of experimentation, we use fingerprint images of size fingerprint image size 80x80 pixels (6400 components). The dataset contains of 168 images obtained from 21 people. The experimental results showed that LDA + SVM gave a classification model that has the highest accuracy at 73.8\%. After using sobel edge detection + LDA + SVM the increased accuracy to $88 \%$. And PCA + SVM, model has the highest accuracy $64.3 \%$ after using sobel edge detection + PCA + SVM model accuracy increased to $88 \%$. The results can be summarized as sobel edge detection when used in conjunction with dimensional reduction technique: principal component analysis or linear discriminant analysis, the recognition of fingerprint image can be significantly enhanced.

\section{References}

(1) Zhou Weina, Han Jun, Zeng Xiaoyang and Yan Weiwei : "Fingerprint verification based on wavelet and edge detection", $9^{\text {th }}$ International Conference on Electronic Measurement \& Instruments, Vol 3, pp. 1001-1004, 2009

(2) G.Sambasiva Rao, C. NagaRaju, Dr.L.S.S. Reddy and Dr.E.V. Prasad : "A Novel Fingerprints Identification System Based on The Edge Detection", Journal of Computer Science and Network Security, Vol. 8, No.12, pp. 394-397, 2008

(3) Wei Cui, Guoliang Wu, Rongjin Hua and HaoYang: "The research of edge detection algorithm for Fingerprint images", Automation Congress (WAC 2008), Vol. 1, pp. 1-5, 2008

(4) Gonzalez, R. C., and Woods, R. E : "Digital Image Processing", Addison-Wesley, 1992

(5) Dinh Minh Nhat, V., and Young Lee, S: "Two dimensional Weighted PCA algorithm for Face Recognition", IEEE International Symposium on Computational Intelligence in Robotics and Automation. pp. 219-223, 2006

(6) Turk, M., and Pentland, A: "Eigenfaces for recognition", Journal of Cognitive Neuronscience. Vol. 3, pp. 71-86, 1991

(7) Balakrishnama, S., and Ganapathiraju, A: "Linear discriminant analysis: a brief tutorial", Mississippi State (MS): Institute for Signal and information Processing, Mississippi State University, 1998

(8) Pornpon Thamrongrat, Ladda Preechaweerakul and Wipada Wetpasit: "Web Page Classification Using Document Frequency and Support Vector Machine", The 12th National Computer Science and Engineering Conference, 2008.

(9) Ali, W., Shamsuddin, S.M., and Ismail A.S: "Web Proxy Cache Content Classification based on Support Vector Machine", Journal of Artificial Intelligence, Vol. 4, No.1, pp. 100-109, 2011 